\title{
Atypischer Hypoadrenokortizismus (Morbus Addison) beim Hund
}

\author{
Ein Fall für internistische Detektivarbeit!
}

Romy M. Heilmann, Roberto B. Heilmann

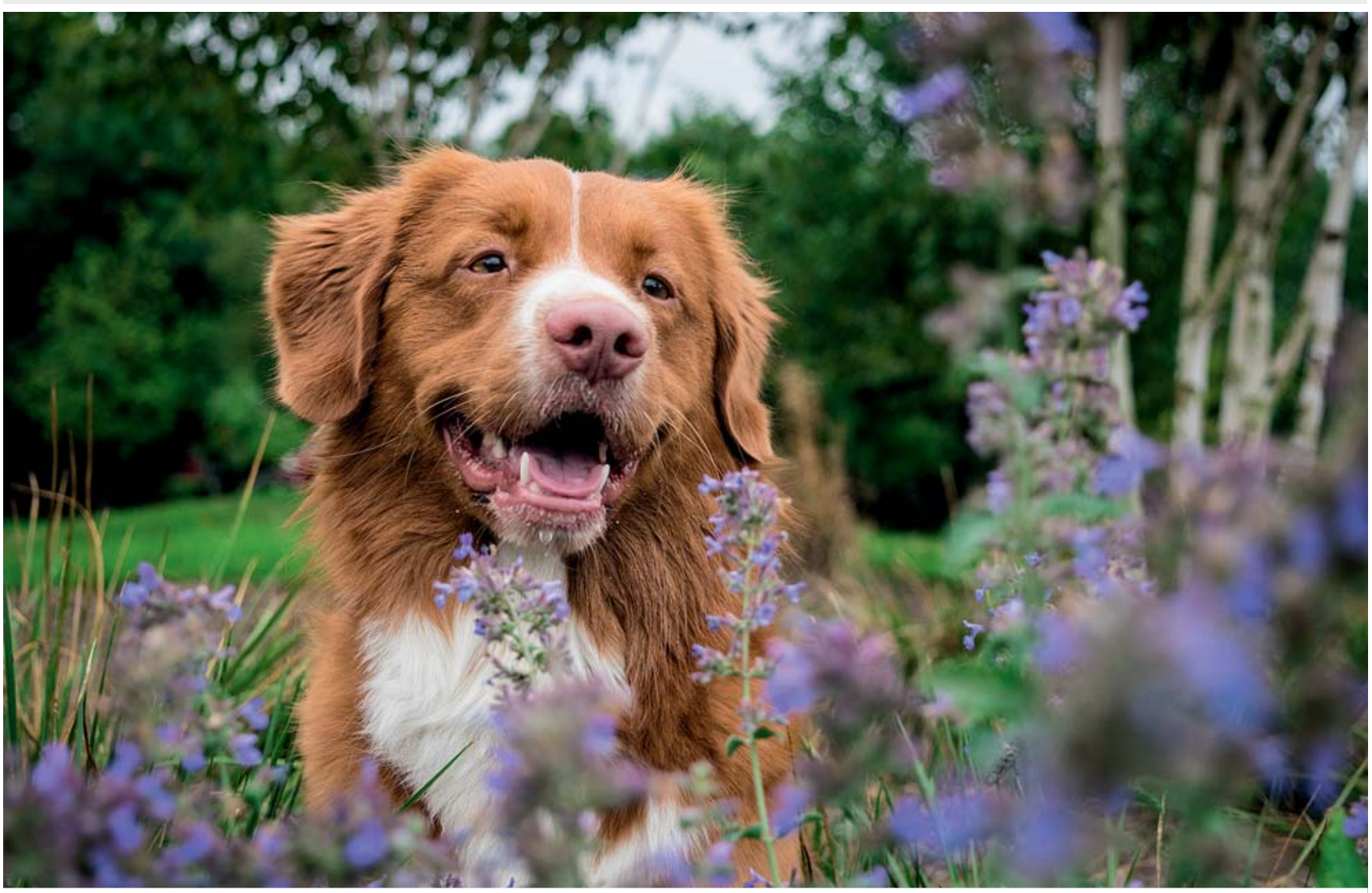

Quelle: @ Iris - stock.adobe.com [rerif]

Wenn die Nebenniere nicht richtig funktioniert, dann zeigt sich das meist mit lehrbuchmäßigen Symptomen. Doch manchmal sind die Krankheitsanzeichen nicht ganz so wegweisend, und die Diagnose wird schwierig. Wir wollen sensibilisieren und zeigen, worauf Sie bei einem atypischen Fall von Hypoadrenokortizismus achten müssen.

\section{Morbus Addison}

Hypoadrenokortizismus (Morbus Addison) beschreibt eine Nebennierenrindeninsuffizienz, die durch eine ungenügende Produktion von Kortikosteroiden und meist auch Mineralokortikoiden gekennzeichnet ist [1]. Im Gegensatz zur typischen Form des Hypoadrenokortizismus, welche infolge der Kortikosteroid- und Mineralokortikoiddefizienz zu einem klinisch und labordiagnostisch relativ charakteristischen Erscheinungsbild führt, stellt die atypische Form des Hypoadrenokortizismus beim Hund auf- grund ihrer Seltenheit und unspezifischen klinischen Symptomatik eine diagnostische Herausforderung dar.

\section{Physiologie der Nebennierenrinde}

Die Bereitstellung von Glukokortikoiden (v.a. Cortisol) und Mineralokortikoiden (Aldosteron) erfolgt durch die Nebennierenrinde. Die Regulation der Cortisolproduktion erfolgt über die Hypophysenvorderlappen-Nebennierenrinden-Achse, wobei eine hohe Cortisolkonzentration im Blut via negativen Feedback-Mechanismus die Adrenocorticotropin (ACTH)-Freisetzung in der Hypophyse so- 


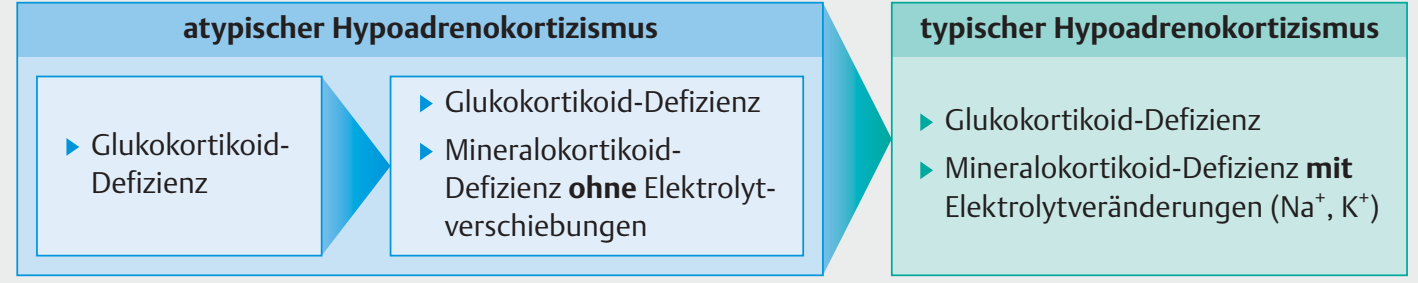

- Abb. 1 Klassifizierung des Hypoadrenokortizismus beim Hund. Nicht aufgezeigt ist die iatrogene Form durch zu schnelles Absetzen exogener Glukokortikoide nach Langzeitgabe bzw. Gabe in hoher Dosis. @ R. Heilmann

wie die Corticoliberin (CRH)-Freisetzung im Hypothalamus hemmt. Da Glukokortikoide eine zentrale Rolle für lebenswichtige Funktionen (Stressantwort, Glukosehomöostase) spielen, kann ein Kortisolmangel durch systemische Hypotension, Hypoglykämie, Schwäche und vermehrte Stressempfindlichkeit auffallen [1,2]. Aldosteron fördert die Rückabsorption von Wasser und Natrium sowie die Ausscheidung von Kalium. Dessen Synthese wird durch Aktivierung des Renin-Angiotensin-Systems (infolge Hyponatriämie, Hypovolämie und systemischer Hypotension) und die Kaliumkonzentration im Plasma bestimmt $[1,2]$.

\section{Nebennierenrindeninsuffizienz}

Die Nebennierenrindeninsuffizienz (Morbus Addison) kennzeichnet sich durch eine zumeist primäre (idiopathische) und nicht adäquate Mineralokortikoid- und/oder Glukokortikoidproduktion. Bei der typischen Form besteht ein Mangel an sowohl Gluko- als auch Mineralokortikoiden, einhergehend mit charakteristischen Elektrolytverschiebungen (Hyperkaliämie, Hyponatriämie) [3].

Seltenere Fälle der atypischen Form zeigen sich entweder mit nur einer Glukokortikoid-Defizienz oder einer Glukokortikoid- und Mineralokortikoid-Defizienz ohne Elektrolytverschiebungen - vermutlich infolge Aldosteron-unabhängiger Kompensationsmechanismen ( $\bullet$ Abb. 1).

Es ist davon auszugehen, dass sich atypische Formen zu einem typischen Hypoadrenokortizismus entwickeln können. Sehr selten führt eine Hypophysenunterfunktion mit ACTH-Defizienz (sekundärer Hypoadrenokortizismus durch Großhirnläsionen wie Neoplasien, Entzündungen oder Trauma) zu einer verminderten Kortisolproduktion in der Nebennierenrinde.

Es können Hunde jeder Rasse und jeden Alters betroffen sein, doch es sind folgende Rasseprädisposition für die Entwicklung eines Hypoadrenokortizismus beschrieben:
Großpudel, Portugiesischer Wasserhund, Nova Scotia Duck Tolling Retriever und Bearded Collie (genetische Ursache) sowie West Highland White Terrier, Cairn Terrier, Deutsche Dogge, Rottweiler, Airedale Terrier, Leonberger, Bernhardiner und Wheaton Terrier (erhöhtes Risiko) [4].

\section{Klinische Symptome}

Hunde mit einem Hypoadrenokortizismus, besonders in atypischer Form, zeigen in der Regel unspezifische klinische Symptome wie Schwäche, Leistungsintoleranz, Inappetenz, Gewichtsverlust bzw. mäßige Körperkondition (niedriger Body Condition Score), Vomitus und Diarrhoe (mitunter hämorrhagisch) [1,2]. Dauer und Schweregrad der klinischen Symptome variieren stark, und sind bei der atypischen Form der Erkrankung aber eher mild ausgeprägt [2,5]. Eine merkliche Dehydratation, hypovolämischer Schock, Bradykardie bzw. -arrhythmie oder Anfälle sind hingegen Befunde, die bei typischer Form des Hypoadrenokortizismus auffallen.

\section{Diagnostik}

Eine minimale Datenbasis (MDB), bestehend aus hämatologischer, blutchemischer und Harnuntersuchung, gibt meist keine spezifischen Befunde zu erkennen. Allenfalls lässt diese den Verdacht eines Hypoadrenokortizismus zu und dient gleichfalls dem Ausschluss anderer Differenzialdiagnosen.

Eine aregenerative normozytäre (seltener mikrozytäre), normochrome Anämie stellt einen unspezifischen Befund dar. Dazu fehlen im Blutbild in der Regel Hinweise auf ein zu erwartendes Stressleukogramm ( $\triangleright$ Kasten S. 94). Insbesondere eine Eosinophilenzahl von $\geq 570 / \mu$ l, eine Lymphozytenzahl von $\geq 750 / \mu l$ sowie ein Neutrophile/Lymphozyten-Quotient (NLQ) von $\leq 2,3$ passen zur Verdachtsdiagnose eines Hypoadrenokortizismus [3,6]. 
STRESSLEUKOGRAMM:

CHARAKTERISTISCHE VERÄNDERUNGEN

- Neutrophilie

- Monozytose

- Eosinopenie

- Lymphopenie

Hypoglykämie, Hypalbuminämie, Hypocholesterinämie und erhöhte Leberenzymaktivitäten im Serum können zu finden sein, ebenso eine prärenale Azotämie. Hyperkaliämie und Hyponatriämie - bzw. ein erniedrigter Natrium/Kalium-Quotient - sind charakteristische blutchemische Veränderungen bei der typischen Form des Hypoadrenokortizismus. Resultierend aus dem Aldosteronmangel, fehlen diese in der Regel bei der atypischen Form $[5,7]$. Auch eine Hypochloridämie, Hyperphosphatämie und Hyperkalzämie finden sich eher bei der typischen Form [1, 2]. Da der Übergang von atypischer Form in typische Form möglich ist, sollten die Elektrolyte bei Patienten mit atypischem Hypoadrenokortizismus regelmäßig kontrolliert werden.

Ein spezifisches Harngewicht $<1,030$ ist ein weiterer Befund bei der typischen Form und steht im Zusammenhang mit der Hyponatriämie.

Interessanterweise können Hunde mit einem Hypoadrenokortizismus auch eine erhöhte Serum-TSH-Konzentration (und erniedrigte Thyroxinwerte) zeigen, die sich im Zuge der Behandlung des Hypoadrenokortizismus normalisieren [8]. Da Hunde allerdings zeitgleich von einem Hypoadrenokortizismus und einer Hypothyreose betroffen sein können, sollte die Serum-TSH-Konzentration kontrolliert werden.

\section{Endokrinologische Diagnostik}

Die Messung des Basalkortisol-Spiegels im Serum eignet sich als Screening-Test. Liegt die Basalkortisolkonzentration bei über $2 \mu \mathrm{g} / \mathrm{dl}(55 \mathrm{nmol} / \mathrm{l})$, so ist ein Hypoadrenokortizismus mit 99-100\%iger Wahrscheinlichkeit ausgeschlossen [9]. Bei einem Basalkortisol-Wert von $<2 \mu \mathrm{g} / \mathrm{dl}$ $(<55 \mathrm{nmol} / \mathrm{l})$ lässt sich die Diagnose durch einen ACTHStimulationstest sichern, wenn die Kortisolkonzentration sowohl vor als auch nach der ACTH-Gabe bei $<2 \mu \mathrm{g} / \mathrm{dl}$ $(<55 \mathrm{nmol} / \mathrm{l})$ liegt ( $\triangleright$ Kasten rechts). In den meisten Fällen misst die Kortisolkonzentration bei einem Hypoadrenokortizismus vor und auch nach ACTH-Gabe unter $1 \mu \mathrm{g} /$ $\mathrm{dl}(<27 \mathrm{nmol} / \mathrm{l})$. Unbedingt auszuschließen ist bei einem solchen Ergebnis die Gabe nebennierenrindensuppressiver Medikamente (z. B. Ketokonazol) bzw. von Glukokortikoiden (einschließlich topisch applizierten, z.B. ophthalmologische Präparate).

Die Messung der endogenen ACTH-Konzentration kann ebenfalls zur Abklärung eines Hypoadrenokortizismus herangezogen werden, insbesondere bei Knappheit an synthetischem ACTH, wobei unbedingt die präanalytischen Anforderungen zu beachten sind. Eine endogene ACTH-Konzentration über dem Referenzintervall (> 50 pmol/l) spricht für einen primären Hypoadrenokortizismus, bei seltenen Fällen der sekundären bzw. tertiären Form ist sie dagegen erniedrigt [3].

Die Plasma-Aldosteronkonzentration, bestimmt vor und nach der Gabe von synthetischem ACTH, kann bei Hunden mit atypischem Hypoadrenokortizismus zur Unterscheidung einer ausschließlichen Glukokortikoid-Defizienz und einer kombinierten Glukokortikoid- und Mineralokortikoid-Defizienz ohne Elektrolytveränderungen herangezogen werden. Letztere Fälle bedingen regelmäßige Elektrolytkontrollen bzw. kann die Gabe eines Mineralokortikoids in Erwägung gezogen werden. Ein Aldosteronmangel lässt sich noch verlässlicher durch ein vermindertes Plasma-Aldosteron:Renin-Verhältnis aufspüren [10]. Allerdings ist die Messung der Renin-Aktivität im Plasma derzeit nicht routinemäßig verfügbar.

\section{Bildgebende Diagnostik}

Die Röntgenuntersuchung von Thorax und Abdomen ist bei Hunden mit einem Hypoadrenokortizismus in der Regel unergiebig, spielt aber eine wichtige Rolle bei der Abklärung anderer Differenzialdiagnosen. Megaösophagus ist ein eher seltener Befund bei Hunden mit Hypoadrenokortizismus [1].

Auch die Sonografie des Abdomens dient der Abklärung weiterer Differenzialdiagnosen (z. B. gastrointestinale Erkrankungen). Die Sonomorphologie der Nebennieren kann mitunter für einen Hypoadrenokortizismus hinweisend sein (z. B. schmale Nebennieren), häufig sind die Nebennieren in der Sonografie aber unauffällig. Des Weiteren eignet sich die sonografische Untersuchung auch für die Abklärung seltener Ursachen des primären Hypoadrenokortizismus (z. B. Neoplasien, Amyloidose, Infarkte oder Blutungen) [11].

\section{DURCHFÜHRUNG EINES}

ACTH-STIMULATIONSTESTS

- Entnahme einer Blutprobe zur Bestimmung der Kortisolkonzentration im Serum (vor ACTH-Injektion)

- Injektion von synthetischem ACTH (Tetracosactid, Synacthen ${ }^{\circledR}$ ) in einer Dosierung von $5 \mu \mathrm{g} /$ kg KG oder $250 \mu \mathrm{g} /$ Hund i. v. oder i. m.

- Entnahme einer 2. Blutprobe im Abstand von $1 \mathrm{~h}$ (nach ACTH-Injektion) zur Bestimmung der stimulierten Kortisolkonzentration im Serum. 
- Tab. 12 Fälle: Hund mit typischem (JULI) und atypischem (WOTAN) Hypoadrenokortizismus.

\begin{tabular}{|c|c|c|}
\hline Patienten & Mischlingshündin JULI. 6 Jahre alt & Boxer-Rüde WOTAN. 91/2 Jahre alt \\
\hline $\begin{array}{l}\text { Vorstellungs- } \\
\text { grund }\end{array}$ & $\begin{array}{l}\text { " akute hochgradige Apathie, Schwäche, Anorexie und Vomitus } \\
\text { " seit } 3 \text { Tagen zunehmende Inappetenz, Gewichtsverlust (ca. } \\
\text { 5\%) }\end{array}$ & $\begin{array}{l}\text { - chronischer Vomitus, z. T. Hämatemese, Inappetenz, Diarrhoe } \\
\text { - Gewichtsverlust (14\% über } 3 \text { Monate) } \\
\text { " intermittierende Apathie }\end{array}$ \\
\hline Anamnese & $\begin{array}{l}\text { - seit } 3 \text { Tagen zunehmende Inappetenz } \\
\text { - Gewichtsverlust (ca. 5\%) } \\
\text { - bereits seit längerem intermittierendes Zittern } \\
\text { " regelmäßig geimpft \& entwurmt } \\
\text { - keine Auslandsanamnese }\end{array}$ & $\begin{array}{l}\text { - ca. } 7 \text { Monate vor Vorstellung bereits Gewichtsabnahme (6\%), } \\
\text { akute Schwächeepisode nach Spaziergang (EKG unauffällig), } \\
\text { kurzzeitige Gabe von Prednisolon (Dosierung: 0,3 mg/kg SID) } \\
\text { mit klinischer Besserung und Körpergewichtszunahme ver- } \\
\text { bunden } \\
\text { - } 4 \text { Monate und } 5 \text { Monate später jeweils } 2 \text { synkopale Anfälle } \\
\text { - vorberichtlich Spondylosen im Bereich der Lumbalwirbelsäule } \\
\text { - regelmäßig geimpft \& entwurmt } \\
\text { - keine Auslandsanamnese }\end{array}$ \\
\hline $\begin{array}{l}\text { Klinische } \\
\text { Unter- } \\
\text { suchung }\end{array}$ & $\begin{array}{l}\text { - Körpergewicht: } 8,7 \mathrm{~kg}, \mathrm{BCS}: 4.5 / 9 \text {, innere Körpertemperatur } \\
\text { (rektal): } 37,5^{\circ} \mathrm{C} \\
\text { - deutlich gestörtes Allgemeinbefinden, dehydriert } \\
\text { - Schleimhäute blass, kapilläre Rückfüllungszeit > } 2 \mathrm{~s} \\
\text { - Herzfrequenz = Pulsfrequenz = } 60 / \text { min } \\
\text { - Atemfrequenz = } 24 / \text { min } \\
\text { - Herzauskultation unauffällig, Auskultation Lunge o. b. B. } \\
\text { - Abdomen palpatorisch weich, nicht dolent } \\
\text { - periphere Lymphknoten palpatorisch unauffällig, rektale } \\
\text { Untersuchung o.b.B. }\end{array}$ & $\begin{array}{l}\text { - Körpergewicht: } 32,8 \mathrm{~kg}, \mathrm{BCS}: 2.5 / 9 \text {, innere Körpertemperatur } \\
\text { (rektal): } 38,3^{\circ} \mathrm{C} \\
\text { - ruhig und aufmerksam, ungestörtes Allgemeinbefinden } \\
\text { - Schleimhäute blassrosa, kapilläre Rückfüllungszeit< } 2 \mathrm{~s} \\
\text { - Herzfrequenz = Pulsfrequenz = } 110 / \text { min } \\
\text { - Atemfrequenz = } 24 / \text { min } \\
\text { - Herzauskultation unauffällig, Auskultation Lunge o.b. B. } \\
\text { - Abdomen palpatorisch weich, nicht dolent, keine Umfangs- } \\
\text { vermehrung } \\
\text { - periphere Lymphknoten palpatorisch unauffällig, rektale } \\
\text { Untersuchung o.b.B. }\end{array}$ \\
\hline $\begin{array}{l}\text { Routine- } \\
\text { diagnostik }\end{array}$ & 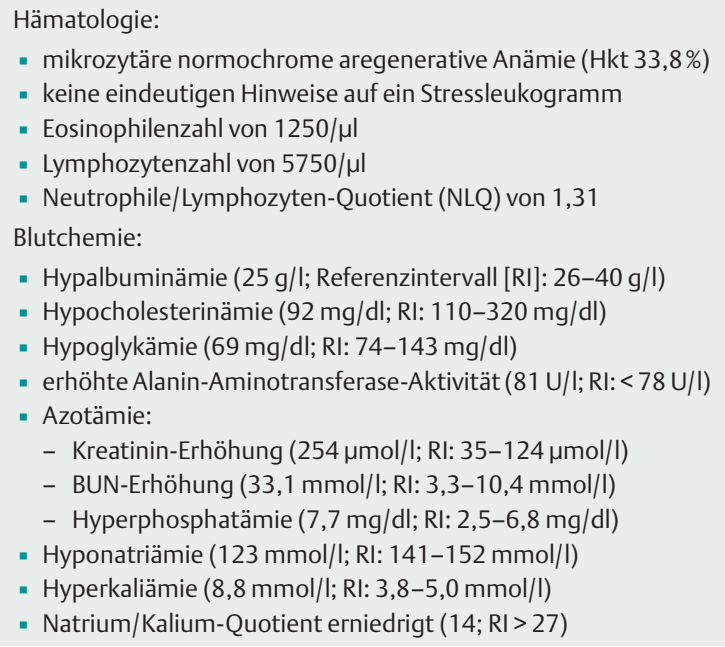 & 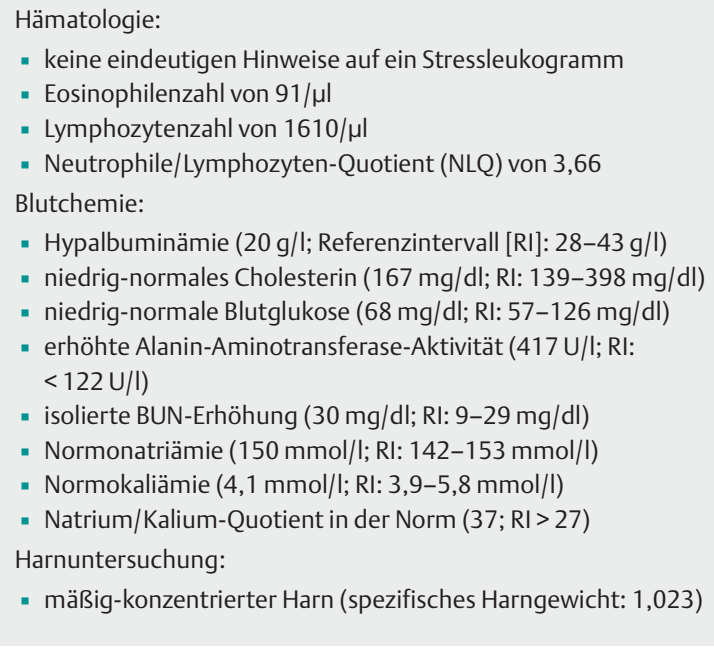 \\
\hline
\end{tabular}


\ab. 12 Fälle: Hund mit typischem (JULI) und atypischem (WOTAN) Hypoadrenokortizismus (Fortsetzung).

\begin{tabular}{|c|c|c|}
\hline Patienten & Mischlingshündin JULI. 6 Jahre alt & Boxer-Rüde WOTAN. 91/2 Jahre alt \\
\hline $\begin{array}{l}\text { Spezielle } \\
\text { Diagnostik }\end{array}$ & $\begin{array}{l}\text { Sonografische Untersuchung Abdomen: } \\
\text { - Nebennieren beidseits deutlich verkleinert }(<0,3 \mathrm{~cm}) \\
\text { positiver Befund im ACTH-Stimulationstest: } \\
\text { - Basalkortisol }<0,1 \mu \mathrm{g} / \mathrm{dl}(\mathrm{Rl}:>2 \mu \mathrm{g} / \mathrm{dl}) \\
\text { - stimulierter Kortisolwert }<0,1 \mu \mathrm{g} / \mathrm{dl}(\mathrm{Rl}:>6 \mu \mathrm{g} / \mathrm{dl})\end{array}$ & 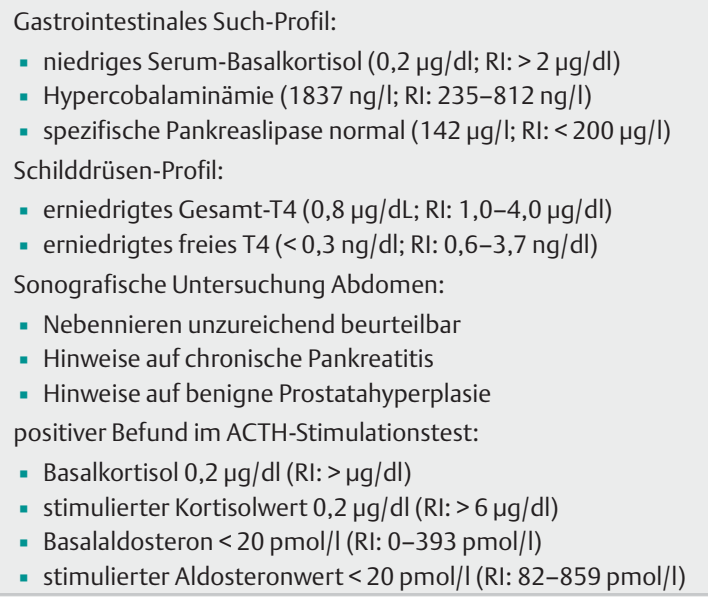 \\
\hline Diagnose & $\begin{array}{l}\text { Hypoadrenokortizismus, typisch (Glukokortikoid- und Minera- } \\
\text { lokortikoiddefizienz, mit Elektrolytverschiebungen), Vorstellung } \\
\text { in Addison-Krise }\end{array}$ & $\begin{array}{l}\text { Hypoadrenokortizismus, atypisch (Glukokortikoid- und Minera- } \\
\text { lokortikoiddefizienz, ohne Elektrolytverschiebungen) }\end{array}$ \\
\hline Therapie & $\begin{array}{l}\text { Notfalltherapie } \\
\text { - Infusionstherapie (Vollelektrolytlösung mit Glukose) } \\
\text { - Antiemese, Gastroprotektion } \\
\text { - Dexamethason einmalig in hoher Dosierung }(0,1 \mathrm{mg} / \mathrm{kg}) \text {, } \\
\text { danach Prednisolon } 0,5 \mathrm{mg} / \mathrm{kg} \text { p. o. für } 5 \mathrm{~d} \\
\left.\text { - DOCP (Zycortal }{ }^{\circledR}\right) 2,2 \mathrm{mg} / \mathrm{kg} \text { s. C. } \\
\text { Erhaltungstherapie: } \\
\text { - Prednisolon } 0,15 \mathrm{mg} / \mathrm{kg} \text { p. o. SID } \\
\text { - DOCP }\left(\text { Zycortal }{ }^{\circledR}\right) \text { s. c. alle } 28 \mathrm{~d}\end{array}$ & $\begin{array}{l}\text { - Prednisolon 7,5 mg p. o. SID (0,23 mg/kg) für 7d, danach: } \\
\text { " Prednisolon 5,0 mg p. o. SID (0,15 mg/kg) für } 5 \text { Monate } \\
\text { " dann Dosisreduktion auf 3,7 mg p. o. SID (0,09 mg/kg) }\end{array}$ \\
\hline Verlauf & $\begin{array}{l}\text { - deutliche und schnelle Besserung der klinischen Symptome } \\
\text { infolge der Notfalltherapie, danach langsame Umstellung auf } \\
\text { Dauertherapieplan } \\
\text { - regelmäßige Kontrolle des Allgemeinbefindens, Körper- } \\
\text { gewichts sowie der Elektrolyte im Blut }\end{array}$ & $\begin{array}{l}\text { - deutliche und schnelle Besserung der klinischen Symptome } \\
\text { nach Beginn der Prednisolon-Gabe } \\
\text { - keine Anfälle oder Schwächeepisoden mehr } \\
\text { - kein Vomitus, keine Diarrhoe, Gewichtszunahme (von 32,5 kg, } \\
\text { BCS 2.5/9 auf 45,5 kg, BCS 6/9; entspricht 29\%) ( A Abb. 2) } \\
\text { - regelmäßige Kontrolle des Allgemeinbefindens, Körper- } \\
\text { gewichts sowie der Elektrolyte im Blut }\end{array}$ \\
\hline $\begin{array}{l}\text { Bemerkun- } \\
\text { gen }\end{array}$ & $\begin{array}{l}\text { Das Vorliegen einer Schocksymptomatik mit Bradykardie (infolge } \\
\text { Hyperkaliämie) ist charakteristisch für eine Addison-Krise. } \\
\text { Die vorhandenen Elektrolytverschiebungen sprachen in diesem } \\
\text { Fall für die typische Form des Hypoadrenokortizismus und mach- } \\
\text { ten sowohl die Bestimmung des Aldosterons als auch der endo- } \\
\text { genen ACTH-Konzentration überflüssig. } \\
\text { Die Therapie der Addison-Krise gestaltet sich zeitgleich mit der } \\
\text { diagnostischen Abklärung. Noch während das Ergebnis des ACTH- } \\
\text { Stimulationstests ausstand, begann die Therapie für die Ver- } \\
\text { dachtsdiagnose Morbus Addison (typische Form). }\end{array}$ & $\begin{array}{l}\text { Die Bestimmung der endogenen ACTH-Konzentration hätte } \\
\text { ebenfalls für die Unterscheidung eines atypischen primären und } \\
\text { eines sekundären Hypoadrenokortizismus herangezogen werden } \\
\text { können, da insbes. bei der atypischen primären Form regelmä- } \\
\text { ßige Elektrolytkontrollen erfolgen sollten, um zu entscheiden, ob } \\
\text { neben der Glukokortikoid- auch eine Mineralokortikoidsubstitu- } \\
\text { tion indiziert ist. }\end{array}$ \\
\hline
\end{tabular}

\section{Therapie}

Im Gegensatz zur typischen Form des Hypoadrenokortizismus, bei welchem Patienten in der Notfallsituation einer akuten Addison-Krise vorgestellt werden können, sind Hunde mit einem atypischen Hypoadrenokortizismus in der Regel klinisch stabil.

Bestehen Hinweise auf eine Dehydratation, sollte jedoch eine dem Patientenzustand angemessene Flüssigkeits- therapie (z. B. mit einer kristalloiden Vollelektrolytlösung wie Ringer-Acetat- oder Ringer-Laktatlösung) erfolgen. Zum Ausgleich einer etwaigen Hypoglykämie wird dem Patienten zusätzlich intravenös Glukose (z. B. Dauertropfinfusion einer mit 2,5\% oder $5 \%$ Glukose angereicherten Vollelektrolytlösung) verabreicht und die Blutglukosekonzentration kontrolliert. Je nach Gesamtzustand des Patienten ist gegebenenfalls eine antiemetische und gastroprotektive Therapie in Erwägung zu ziehen. 


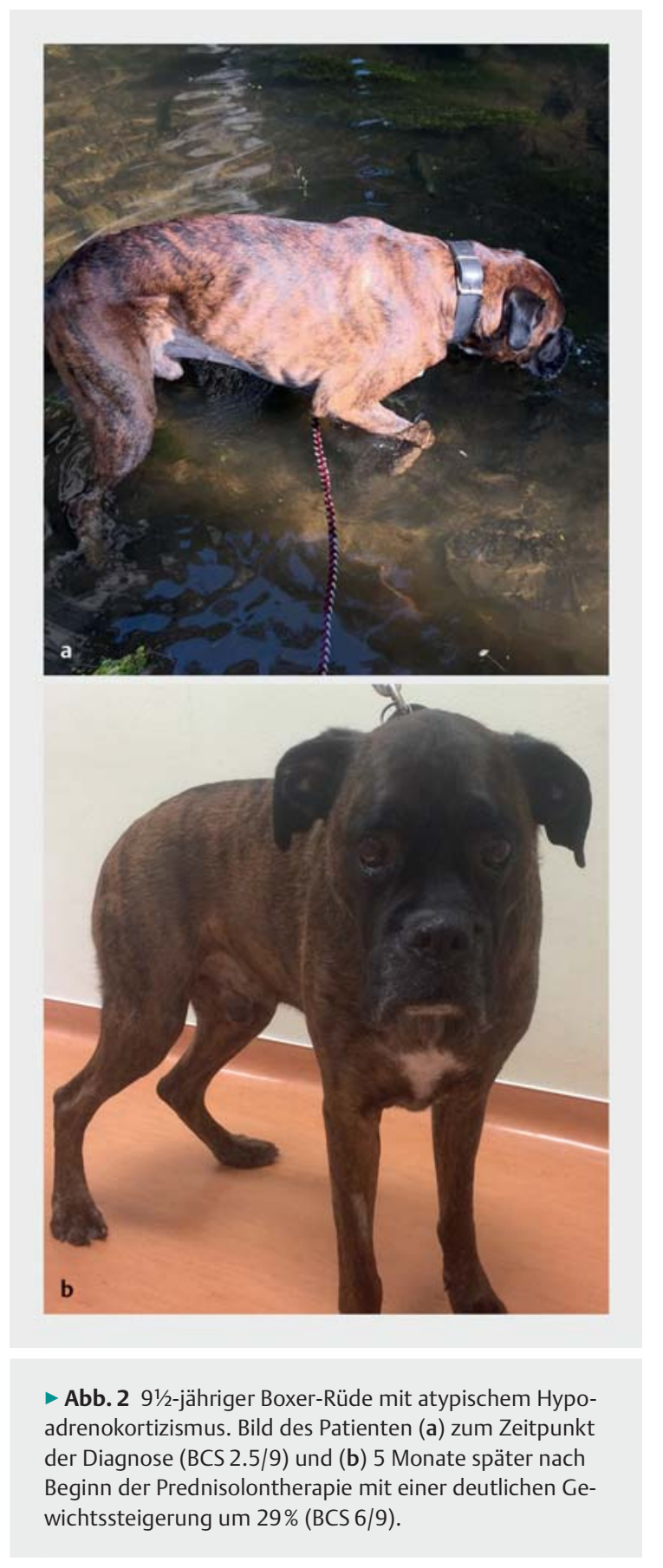

\section{Glukokortikoidsubstitution}

Nach der Durchführung des ACTH-Stimulationstests bzw. Vorliegen der entsprechenden Resultate sollte die Glukokortikoidsubstitution begonnen werden, wobei diese lebenslang notwendig ist! Dabei ist Prednisolon das Präparat der Wahl beim Hund, während schneller wirksame Glukokortikoide (z.B. Dexamethason, Hydrocortison) eher in der Therapie einer Addison-Krise eingesetzt werden. Begonnen wird die Therapie mit Prednisolon über einige Tage in etwas höherer Dosierung $(0,3-0,5 \mathrm{mg} / \mathrm{kg}$ p.o. SID-BID), gefolgt von einer graduellen Reduktion 
hin zur physiologischen Prednisolondosis $(0,05-0,2 \mathrm{mg} /$ kg p.o. SID) [1,2]. Diese niedrigst-wirksame Prednisolondosis wird für die Langzeittherapie fortgeführt (Erhaltungstherapie) und bei zu erwartenden Stresssituationen (z. B. elektiver Eingriff) kurzzeitig erhöht (um 100-300\%) $[1,2]$.

\section{Mineralokortikoidsubstitution}

Während die Diagnose eines typischen Hypoadrenokortizismus mit Elektrolytverschiebungen (Hyponatriämie, Hyperkaliämie) in jedem Fall eine Mineralokortikoidsubstitution mit regelmäßiger Kontrolle der Elektrolyte erfordert, ist die Mineralokortikoid-Gabe beim atypischen Hypoadrenokortizismus abhängig vom endogenen Mineralokortikoidstatus zu entscheiden.

Hunde mit einem atypischen Hypoadrenokortizismus ohne Elektrolytverschiebungen und mit normalem Plasma-Aldosteronspiegel sollten zunächst nur eine Glukokortikoidsubstitution erhalten, die Elektrolyte aber regelmäßig kontrolliert werden. Bei Hunden mit einem atypischen Hypoadrenokortizismus ohne Elektrolytveränderungen jedoch einer nachgewiesenen Mineralokortikoid-Defizienz (niedriger Plasma-Aldosteronspiegel) sollte entweder eine Mineralokortikoidsubstitution (Desoxycortonpivalat, DOCP: Zycortal ${ }^{\circledR}$, Anfangsdosis 1,5$2,2 \mathrm{mg} / \mathrm{kg}$ s.c. alle 25-28 Tage [12]; Percorten ${ }^{\circledR}-\mathrm{V}$, Anfangsdosis $2,2 \mathrm{mg} / \mathrm{kg}$ i.m. alle 25-28 Tage) erwogen oder die Elektrolyte engmaschig kontrolliert werden (anfangs alle 2-4 Wochen, später ggf. mit etwas längerem Abstand) [1,2].

\section{Therapiekontrolle und Prognose}

Glukokortikoid-Nebenwirkungen (Polyphagie, Polydipsie/ Polyurie, Körpergewichtszunahme, Hecheln, Haut- und Fellveränderungen) können mitunter bereits bei sehr niedriger Dosierung auftreten und eine Dosisreduktion (um ca. 10-15\%) erfordern. Im Gegensatz dazu sollten Hinweise auf eine ungenügende Glukokortikoidsubstitution (Apathie, Schwäche, Hypo- oder Anorexie, Vomitus, Diarrhoe) Anlass zu einer Dosiserhöhung (um ca. 50\%) sein.

Erfolgt eine Mineralokortikoidsubstitution, sind in der Therapiekontrolle (anfangs alle 2-4 Wochen, später alle 1-3 bzw. bei guter Einstellung alle 3-6 Monate) weiterhin die Elektrolytkonzentrationen (Natrium, Kalium) und der systemische Blutdruck relevant. Findet sich 10-14 Tage nach der DOCP-Gabe eine Hypokaliämie bzw. eine Hypernatriämie, sollte die nächste DOCP-Dosis um 10-20\% reduziert werden. Bestehen diese Elektrolytverschiebungen noch zum Zeitpunkt der nächsten DOCP-Injektion, ist diese zunächst auszusetzen und die Elektrolyte 57 Tage später zu kontrollieren, um neben einer Dosisreduktion (um 10-20\%) auch über ein längeres Dosierungsintervall zu entscheiden. Die Entwicklung einer sys- temischen Hypertension (d.h. systolischer Blutdruck $>140 \mathrm{mmHg}$ ) bedingt ebenso - je nach Ausmaß - eine DOCP-Dosisreduktion oder Beendigung der Mineralokortikoidsubstitution. Polyurie/Polydipsie, meist als Prednisolon-Nebenwirkung interpretiert, können auch klinische Symptome einer DOCP-Überdosierung darstellen.

Bei adäquater Therapie (d. h. Glukokortikoid- und ggf. Mineralokortikoidsubstitution) und regelmäßiger Kontrolle ist der primäre Hypoadrenokortizismus in der Regel mit einer sehr guten Langzeitprognose verbunden, indem betroffene Hunde bei guter Lebensqualität eine normale Lebenserwartung haben $[1,2]$.

\section{Fazit}

Hunde mit einem typischen Hypoadrenokortizismus sind klinisch und labordiagnostisch in der Regel offensichtlich, während die diagnostische Abklärung von Patienten mit atypischem Hypoadrenokortizismus „internistische Detektivarbeit" verlangt und Tierärzte sich dafür erst sensibilisieren müssen. Doch die gute Nachricht ist: Beide Formen des Hypoadrenokortizismus sind bei guter Besitzercompliance mit einer sehr guten Langzeitprognose verbunden!

\section{Korrespondenzadresse}

Prof. Dr. Romy M. Heilmann

Klinik für Kleintiere

Veterinärmedizinische Fakultät der Universität Leipzig

An den Tierkliniken 23

04103 Leipzig

romy.heilmann@kleintierklinik.uni-leipzig.de

Roberto B. Heilmann

Tierarztpraxis Heilmann

Nimschützer Str. 7

02625 Bautzen

Literatur

[1] Kalenyak K, Heilmann RM. Canine hypoadrenocorticism - an update on pathogenesis, diagnosis and treatment. Tierarztl Prax Ausg K Kleintiere Heimtiere 2018; 46(3): 163-175

[2] Lathan P, Thompson AL. Management of hypoadrenocorticism (Addison's disease) in dogs. Vet Med (Auckl) 2018; 9: 1-10

[3] Zeugswetter FK, Schwendenwein I. Diagnostic efficacy of the leukogram and the chemiluminometric ACTH measurement to diagnose canine hypoadrenocorticism. Tierarztl Prax Ausg K Kleintiere Heimtiere 2014; 42(2): 223-230

[4] Hanson JM, Tengvall K, Bonnett BN et al. Naturally occurring adrenocortical insufficiency - an epidemiological study based on a Swedish-insured dog population of 525,028 dogs. J Vet Intern Med 2016; 30: 76-84

[5] Thompson AL, Scott-Moncrieff JC, Anderson JD. Comparison of classic hypoadrenocorticism with glucocorticoid-deficient hypoadrenocorticism in dogs: 46 cases (1985-2005). J Am Vet Med Assoc 2007; 230: 1190-1194 
[6] Seth M, Drobatz KJ, Church DB et al. White blood cell count and the sodium to potassium ratio to screen for hypoadrenocorticism in dogs. J Vet Intern Med 2011; 25: 1351-1356

[7] Richartz J, Neiger R. Hypoadrenokortizismus ohne klassische Elektrolytveränderungen bei sieben Hunden. Tierärztl Prax 2011; 39 (K): 163-169

[8] Reusch CE, Fracassi F, Sieber-Ruckstuhl NS et al. Altered serum thyrotropin concentrations in dogs with primary hypoadrenocorticism before and during treatment. J Vet Intern Med 2017; 31: 1643-1648

[9] Lennon EM, Boyle TE, Hutchins RG et al. Use of basal serum or plasma cortisol concentrations to rule out a diagnosis of hypoadrenocorticism in dogs: 123 cases (2000-2005). J Am Vet Med Assoc 2007; 231: 413-416

[10] Javadi S, Galac S, Boer P et al. Aldosterone-to-renin and cortisol-to-adrenocorticotropic hormone ratios in healthy dogs and dogs with primary hypoadrenocorticism. J Vet Intern Med 2006; 20: 556-561

[11] Labelle P, De Cock HE. Metastatic tumors to the adrenal glands in domestic animals. Vet Pathol 2005; 42: 52-58

[12] Sieber-Ruckstuhl NS, Reusch CE, Hofer-Inteeworn NE et al. Evaluation of a low-dose desoxycorticosterone pivalate treatment protocol for long-term management of dogs with primary hypoadrenocorticism. J Vet Intern Med 2019; 33: 12661271

Bibliografie

DOI https://doi.org/10.1055/a-0922-8588

Veterinärspiegel 2019; 29: 92-99

(c) Georg Thieme Verlag KG Stuttgart · New York

ISSN 0940-8711 